\title{
Evaluation of an in Situ Polymerizing Hydrogel Applied in Tumor Excision Cavities during Breast Conservation Surgery
}

\author{
M. Korell ${ }^{1}$, F. Brassel ${ }^{2}$, G. Bilir ${ }^{3}$, J. Pagels ${ }^{3}$ \\ ${ }^{1}$ Department of OBGYN, Johanna Etienne-Krankenhaus, Neuss, Germany \\ ${ }^{2}$ Department of Radiology, Klinikum Duisburg, Duisburg, Germany \\ ${ }^{3}$ Department of OBGYN, St. Josef Krankenhaus, Moers, Germany \\ Email:m.korell@freenet.de
}

Received September 14, 2013; revised October 12, 2013; accepted October 20, 2013

Copyright (C) 2013 M. Korell et al. This is an open access article distributed under the Creative Commons Attribution License, which permits unrestricted use, distribution, and reproduction in any medium, provided the original work is properly cited.

\begin{abstract}
Study Objective: To evaluate the imaging characteristics and healing following application of an absorbable biocompatible hydrogel into the excision cavity following breast conservation surgery. Design: Non-randomized study evaluating hydrogel application feasibility, post-operative hydrogel imaging characteristics, cavity healing following hydrogel absorption and the resulting breast cosmesis. Setting: Teaching hospital University of Essen, Germany. Patients: Seven (7) patients underwent unilateral or bilateral breast conservation surgery to remove cancerous or benign tumors. Interventions: All patients received hydrogel application following primary tumor excision. Imaging performed in the first postoperative week included ultrasound, MRI and CT. Breast cosmesis scores were obtained at one and three months following surgery. Measurements and Main Results: While preliminary, a relationship between percent cavity filling and drainage was observed, with $30 \%$ or more cavity filling resulting in decreased drainage. Additionally, the hydrogel was readily visible under ultrasound, MRI and CT imaging modalities. Finally, patients were satisfied and surgeons felt that patient cosmesis was improved relative to their prior experience. Conclusion: Initial data suggest that hydrogel filling may reduce excision cavity drainage, which may reduce seroma or hematoma formation. Additionally, these gels may improve cavity visibility and stability, furthering the use of partial breast irradiation. Finally, while more studies are required, these materials may have a role in improving long term patient cosmesis.
\end{abstract}

Keywords: Lumpectomies; Breast Radiation; Seroma Prevention; Breast Cosmesis

\section{Introduction}

The current standard of care for the surgical treatment of patients diagnosed with breast cancer is breast conservation surgery (BCS, frequently called lumpectomy) whenever it is possible. The BCS procedure involves removal of the breast cancer along with a margin of normal breast tissue, followed routinely by whole breast radiation therapy (WBRT) to eliminate potential tumor cells beyond the resected margins. In addition to preserving much of the patient's breast tissue, this technique has been shown to be as efficacious as mastectomy, with similar survival rates over 15 years of follow up [1-4].

For further reduction of local breast cancer recurrence, a radiation boost is applied to the tumor bed, which is guided by using metal clips as marker. This approach is justified since cancer recurrence is highest near the excision site, with $86 \%$ of recurrences in the same quadrant as the excision, as shown in one multicenter study [5]. However, unlike many other radiation oncology targets (i.e. prostate), the margins of the excised cavity are often difficult to visualize following surgery, as demonstrated by large inter-observer differences while attempting to delineate and contour the cavities [6-8]. Surgical clips are often used to mark discreet points within the cavity, but the vast majority of the cavity margins remain unmarked, and often difficult to delineate. Frequently seromas and/or hematomas form within the excision cavities following surgery, assisting in cavity visualization, but seroma formation is an unpredictable complication of BCS and therefore is not a reliable aid for visualization.

Further complicating cavity targeting is the change in cavity volume due to seroma absorption in the months following surgery. Several researchers reported changes in mean excision cavity volumes during radiation therapy 
ranging from $22.5 \%$ to $54 \%$ [9-13]. Cavity volume change during radiation can result in movement of normal tissue into the radiation treatment volume, or worse, movement of the cavity margins, potentially containing residual tumor cells, out of the radiation treatment volume.

A marker that allows for improved post-operative identification and localization of the excision cavity margins while preserving the cavity volume through the course of radiation therapy would overcome a number of the challenges related to partial breast irradiation. Further, stable and visible cavity margins would facilitate accelerated partial breast irradiation (APBI), which has been reported to be about half the cost of WBRT [14].

The aim of this study is to gather initial clinical data on the use of in situ polymerizing polyethylene (PEG) hydrogel instilled into lumpectomy cavities to facilitate postoperative cavity visibility via ultrasound, MR and CT imaging. Additional study endpoints include patient comfort and tolerance of implant, breast drainage, physician scored cosmesis at 1 and 3 months following implantation, and collection of adverse events experienced by patients.

\section{Materials and Methods}

Following institutional Ethics Committee protocol approval and receipt of Informed Consent, a total of 7 patients were enrolled in this evaluation. Patient ages ranged from 19 to 75 years (mean $=47$ years), and 4 of the patients had benign disease, while 3 patients had been previously diagnosed with breast cancer. 6 of the patients had unilateral procedures, while one patient had bilateral lumpectomies, resulting in 8 hydrogel instillations. Tumor volumes ranged from 1 to $165 \mathrm{cc}$, with a mean volume of $63 \mathrm{cc}$.

Following completion of tumor excision and confirmation of cavity hemostasis, one drainage catheter was tunneled into the cavity, and one silicone instillation catheter was introduced into the excision cavity via the surgical incision. With the drainage catheter clamped to prevent plugging, between 4 to $20 \mathrm{ml}$ of hydrogel (mean $=14 \mathrm{ml}$ ) was instilled via the silicone catheter, which was withdrawn from the cavity prior to hydrogel polymerization (Figure 1). All but one of the cavities were partially closed with sutures prior to injection, and depending on incision location, half of the skin incisions (4 of 8 ) were mostly closed prior to hydrogel injection to prevent loss of liquid hydrogel precursor while the hydrogel polymerized.

The hydrogel used in this evaluation was a polyethylene glycol (PEG) based hydrogel initially approved in Europe for use as a dura mater sealant, but subsequently approved for use on the lung, in vascular surgery and for the prevention of peridural adhesions following spinal

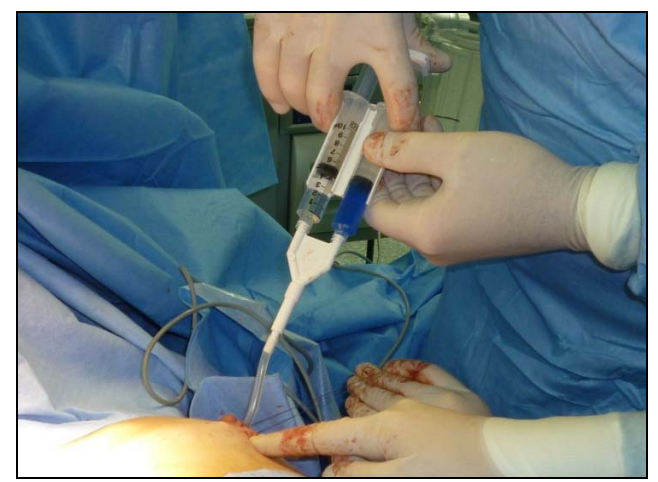

Figure 1. Injection of hydrogel precursors into the lumpectomy cavity, where they polymerized forming a soft, absorbable hydrogel.

surgery (Confluent Surgical, Bedford, MA, USA). Following mixing of the two precursor solutions, a reaction between end-functionalized PEG and trilysine results in polymerization, or creation of a soft, solid hydrogel, within seconds without producing measureable heat. The resulting hydrogel contains hydrolysable linkages at each PEG-trilysine junction, causing the hydrogel to break down over time and be absorbed. For this application both precursors were diluted 1:1 with sterile saline prior to applicator assembly using a commercially available mixing Y-connector (Micromedics, Inc. St. Paul, MN, USA). This dilution slightly slowed down the polymerization rate reducing the likelihood of applicator plugging during instillation, and reduced the absorption time to within 1 month (as demonstrated by prior testing in $37^{\circ} \mathrm{C}$ PBS). While the relatively short persistence of this material will not make it an ideal candidate for partial breast radiation targeting, it will still provide valuable information regarding hydrogel visualization, injection feasibility, patient tolerance, cosmesis, and could potentially benefit the patient by reducing drainage and the likelihood of problematic seromas.

Following surgery ultrasound (US) images of cavities were obtained within 2 days, and drains were removed between 1 and 5 days (mean $=3$ days), with the total drainage recorded. In addition to US images, magnetic resonance (MRI) or computed tomography (CT) images were obtained within one week of surgery. At the 1 month postoperative visit the surgeon queried patients regarding postoperative pain, and scored both postoperative pain and treated breast cosmesis as either worse than expected (score of -1 ), as expected ( 0 ) or better than expected (1, relative to hospital experience). At 3 months following surgery the treated breast cosmesis was scored as before, along with a patient's estimation of breast cosmesis score using the visual analogue scale (VAS), with a range from 0 (completely unsatisfied) to 10 (very satisfied).

All three of the breast cancer patients received postop- 
erative chemotherapy and whole breast radiation.

\section{Results}

The course of healing for all patients was normal, although one patient experienced a superficial wound infection that resolved within three weeks without further treatment. Overall the postoperative pain was scored as either less than normal, or normal to the surgeon's clinical experience.

Breast drainage ranged from 5 to $180 \mathrm{ml}($ mean $=59$ $\mathrm{ml}$, median $=8 \mathrm{ml}$ ). Of note, $94 \%$ of the total study drainage came from the three patients with the largest lumpectomies. In the other breasts drainage averaged 6 ml. A sharp drop in drainage was noted when the gel volume was greater than $30 \%$ of the cavity volume (Figure 2).

In every patient the hydrogel was readily apparent under US imaging. Initially the hydrogel had a hypointense appearance, like water. Over time gel absorption was observed (Figure 3).

The gel was found to be readily visible using MR imaging, as seen in Figure 4. The gel is most conspicuous using T2 with fat suppression, although it is visible under all modalities. In cases with low drainage the cavity had a much more uniform density, suggesting a better cavity fill and less fluid collection (as seen in Figure 4(a)). In a patient that exhibited $180 \mathrm{ml}$ of drainage (with $12 \%$ cavity filling), different densities are visible within the cavity suggesting both fluid and gel collections, as seen in Figures 4(c) and (d).

The hydrogel was also visible under CT imaging at one week following implantation, with a good agreement between US and CT cavity shapes (Figure 5).

Patients satisfaction with the outcome at 3 months postoperatively was high: mean $=8.8(\min 8-\max 9.5)$.

Surgeon scored breast cosmesis at one and three months following surgery was scored as "Better than expected" $(50 \%, 62.5 \%)$, "As expected" (50\%, 25\%) and "Worse than expected" $(0 \%, 12.5 \%)$, respectively. Using the scoring method outlined earlier, this represents a $50 \%$

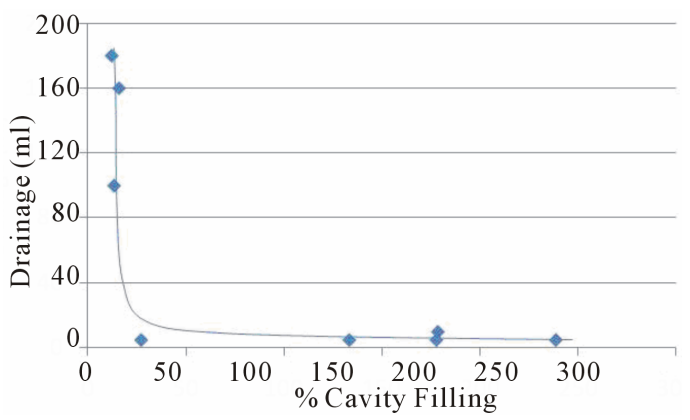

Figure 2. Postoperative drainage was observed to drop when the gel volume was at least $30 \%$ of the lumpectomy cavity volume.

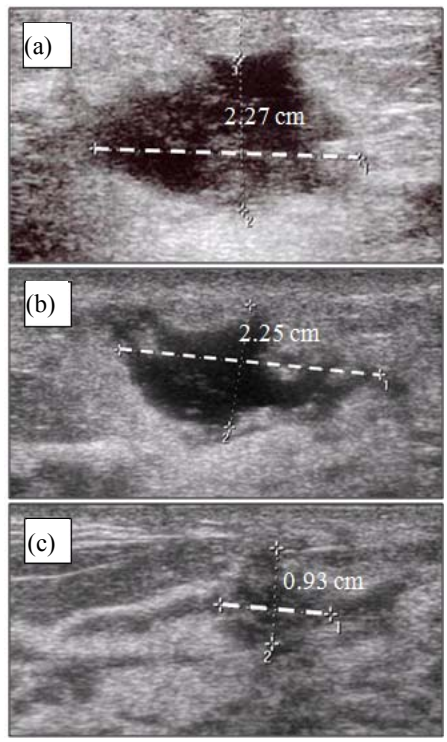

Figure 3. Hydrogel appearance under ultrasound imaging in a single patient at 1 day (a), 1 week (b), and at 14 weeks (c) following implantation.
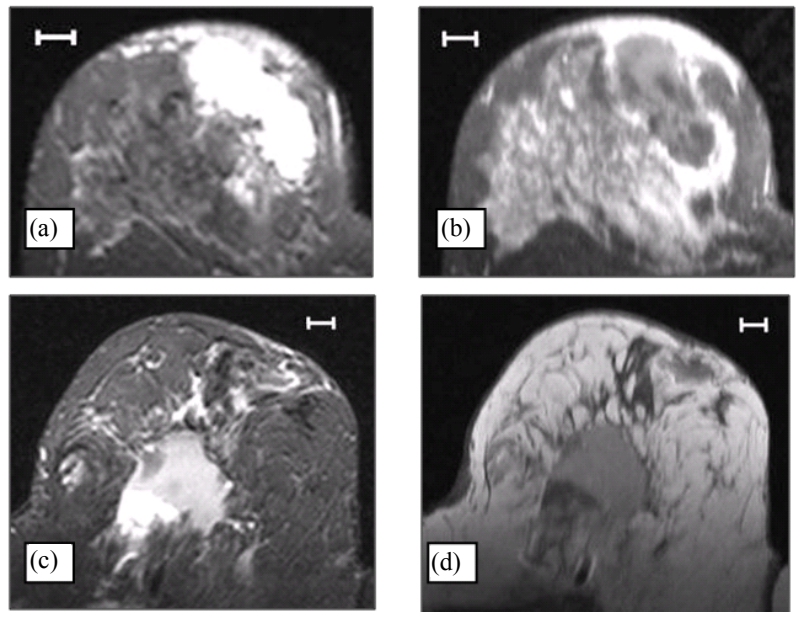

Figure 4. Six days following implantation the gel is clearly visible under MRI using fat suppression with both T2 (a), and T1 with contrast (b). Differing densities within the cavity of one patient suggested both fluid and gel collections at 7 days with $\mathrm{T} 2$ and fat suppression (c), and with $\mathrm{T} 1$ and no fat suppression (d). Scale $=1 \mathrm{~cm}$.

improvement in cosmetic outcome at both time points, relative to prior surgeon experience (Figure 6).

\section{Discussion}

Some of the patient care challenges following breast conservation surgery include 1) avoiding the formation of symptomatic seroma or hematoma, 2) accurate identification of the tumor cavity margins for targeted radiotherapy during either PBI or boost procedures, 3) the change in cavity volume over time, also limiting the potential use of PBI, 4) the long term cosmetic result due to 


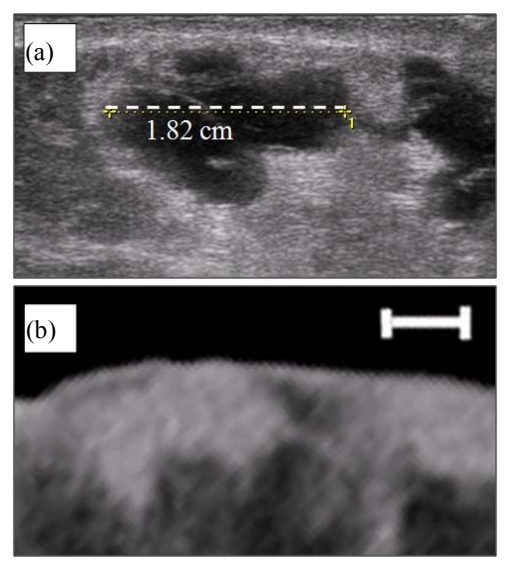

Figure 5. Close agreement between ultrasound image (a) and CT image (b) in patient one week following surgery. Scale $=1 \mathrm{~cm}$.

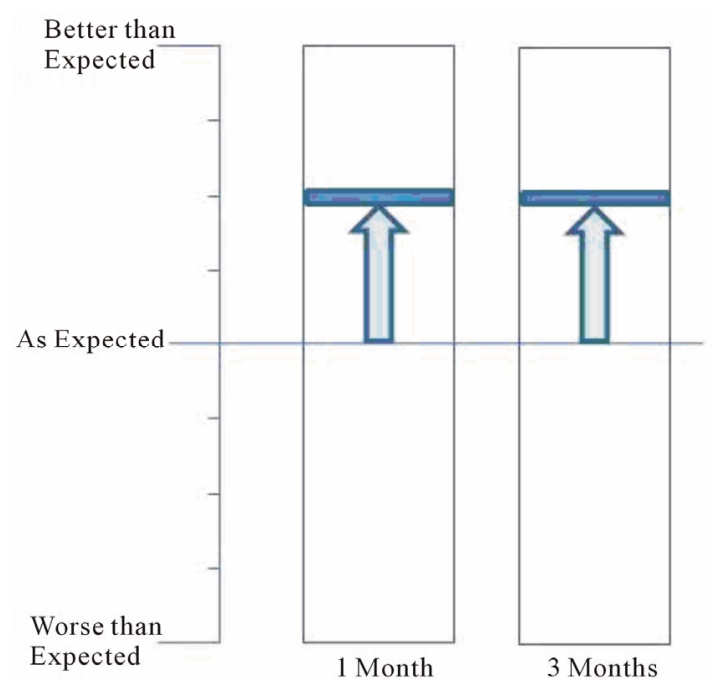

Figure 6. Surgeon scored breast cosmesis one and three months following surgery showed a $50 \%$ improvement relative to prior surgeon experience.

volume loss and scar tissue retraction, and 5) impact on tissue visualization during subsequent diagnostic imaging procedures (mammography) due to scar formation.

The hydrogel used in this evaluation has been approved and used as a sealant and adhesion barrier in different areas of the body. As such, these features may have a role in improving outcomes following BCS. These materials have the potential to reduce the incidence of postoperative seroma and hematoma formation by both acting as a cavity filler, and by sealing the cavity walls to prevent fluid migration into the cavity. While limited in sample size, the data from this preliminary study suggest that when at least $30 \%$ of the cavity volume is replaced with hydrogel that seals the cavity margins, postoperative drainage, and presumably postoperative seroma formation, may be reduced.

Additionally, while the hydrogel utilized for this evaluation will likely not last long enough for PBI, it may provide adequate cavity delineation and stability for accelerated PBI. Imaging with both MRI and CT demonstrated hydrogel visibility, leaving open the possibility of contouring the hydrogel during radiation treatment planning. Indeed, recent studies in cadavers with similar cavity filling hydrogels demonstrates that these materials can be contoured with $\mathrm{CT}$, and if a $15 \mathrm{~mm}$ treatment margin is utilized due to improved cavity visibility, then the total radiation dose to the normal breast tissue, heart and lungs can be reduced [15].

Another potential benefit of hydrogel excision cavity fillers is an improvement in patient cosmesis. Permanent fillers could result in long term volume replacement, preventing contour deficits. Absorbable fillers, while not providing long term volume replacement, may also improve cosmesis by allowing tissue filling of the cavity during absorption, or by reducing the scar formation and subsequent scar retraction which can lead to scar invagination.

While this evaluation is very preliminary, it does demonstrate some of the potential advantages on excision cavity filling with hydrogels at the time of surgery. These materials have the potential to reduce seroma formation, improve cavity visualization and delineation, and to improve long term patient cosmesis. These results warrant further clinical research of this technique.

\section{Acknowledgements}

The authors would like to thank Alex Norbash, MD for assistance in interpreting the MR and CT images.

\section{REFERENCES}

[1] W. C. Dooley, "Breast Cancer: Surgical Therapy," In: J. L. Cameron, Ed., Current Surgical Therapy, 3rd Edition, Mosby, St. Louis, 1995.

[2] B. Fisher, S. Anderson, C. K. Redmond, et al., "Reanalysis and Results after 12 Years of Follow-Up in a Randomized Clinical Trial Comparing Total Mastectomy with Lumpectomy or without Irradiation in the Treatment of Breast Cancer," New England Journal of Medicine, Vol. 333, No. 22, 1995, pp. 1456-1461. http://dx.doi.org/10.1056/NEJM199511303332203

[3] B. Fisher, M. Bauer, R. Margolese, et al., "Five-Year Results of a Randomized Clinical Trial Comparing Total Mastectomy and Segmental Mastectomy with or without Radiation in the Treatment of Breast Cancer," New England Journal of Medicine, Vol. 312, No. 11, 1985, pp. $665-673$. http://dx.doi.org/10.1056/NEJM198503143121101

[4] J. A. Jacobson, O. N. Danforth, K. H. Cowan, et al., "Ten-Year Results of a Comparison of Conservation with Mastectomy in the Treatment of Stage I and II Breast Cancer," New England Journal of Medicine, Vol. 332, No. 14, 1995, pp. 907-911. 
http://dx.doi.org/10.1056/NEJM199504063321402

[5] B. Fisher, S. Anderson, J. Bryant, et al., "Twenty-Year Follow-Up of a Randomized Trial Comparing Total Mastectomy, Lumpectomy, and Lumpectomy plus Irradiation for the Treatment of Invasive Breast Cancer," New England Journal of Medicine, Vol. 347, 2002, pp. 1233- 1241. http://dx.doi.org/10.1056/NEJMoa022152

[6] C. Hurkmans, M. Admiraal, M. van der Sangen, et al., "Significance of Breast Boost Volume Changes during Radiotherapy in Relation to Current Clinical Interobserver Variations," Radiotherapy \& Oncology, Vol. 90, No. 1, 2009, pp. 60-65. http://dx.doi.org/10.1016/j.radonc.2007.12.001

[7] R. P. Petersen, P. T. Truong, H. A. Kader, et al., "Target Volume Delineation for Partial Breast Radiotherapy Planning: Clinical Characteristics Associated with Low Interobserver Concordance," International Journal of Radiation Oncology • Biology • Physics, Vol. 69, No. 1, 2007, pp. 41-48.

[8] H. Struikmans, C. Warlam-Rodenhuis, T. Stam, et al., "Interobserver Variability of Clinical Target Volume Delineation of Glandular Breast Tissue and of Boost Volume in Tangential Breast Irradiation," Radiotherapy \& Oncology, Vol. 76, No. 3, 2005, pp. 293-299. http://dx.doi.org/10.1016/j.radonc.2005.03.029

[9] T. W. Flannery, E. M. Nichols, S. B. Cheston, et al., "Repeat Computed Tomography Simulation to Assess Lumpectomy Cavity Volume during Whole-Breast Irradiation," International Journal of Radiation Oncology • Biology •
Physics, Vol. 75, No. 3, 2009, pp. 751-756.

[10] G. Jacobson, V. Betts and B. Smith, "Change in Volume of Lumpectomy Cavity during External-Beam Irradiation of the Intact Breast," International Journal of Radiation Oncology • Biology • Physics, Vol. 65, No. 4, 2006, pp. 1161-1164.

[11] K. S. Oh, F. M. Kong, K. A. Griffith, et al., "Planning the Breast Tumor Bed Boost: Changes in the Excision Cavity Volume and Surgical Scar Location after Breast-Conserving Surgery and Whole-Breast Irradiation," International Journal of Radiation Oncology • Biology • Physics, Vol. 66, No. 3, 2006, pp. 680-686.

[12] R. Sharma, M. Spierer, S. Mutyala, et al., "Change in Seroma Volume during Whole-Breast Radiation Therapy," International Journal of Radiation Oncology • Biology • Physics, Vol. 75, No. 1, 2009, pp. 89-93.

[13] T. J. Yang, P. H. Elkuizen, R. T. Minkema, et al., "Clinical Factors Associated with Seroma Volume Reduction in Breast-Conserving Therapy for Early-State Breast Cancer: A Multi-Institutional Analysis," International Journal of Radiation Oncology • Biology • Physics, Vol. 76, No. 5, 2010, pp. 1325-1332.

[14] A. G. Taghian and C. Bourgier, "Counterpoint: Conformal External Beam for Accelerated Partial Breast Irradiation," Brachytherapy, Vol. 8, No. 2, 2009, pp. 184-188.

[15] R. C. Susil, E. C. Ford, M. S. Camp, et al., "Hydrogel Fillers May Improve Visibility of Breast Lumpectomy Cavities," Submitted for Publication. 